\title{
Gastric neuroendocrine tumors: management and challenges
}

This article was published in the following Dove Press journal:

Gastrointestinal Cancer:Targets and Therapy

29 September 2017

Number of times this article has been viewed

\section{Daniel H Kwon' \\ Eric K Nakakura ${ }^{2}$ \\ Emily K Bergsland ${ }^{3}$ \\ Sun-Chuan Dai ${ }^{4}$}

'Department of Medicine, ${ }^{2}$ Division of General Surgery, ${ }^{3}$ Division of Hematology/Oncology, ${ }^{4}$ Division of Gastroenterology, University of California San Francisco, San Francisco, CA, USA
Correspondence: Sun-Chuan Dai Division of Gastroenterology, San Francisco General Hospital, I00I Potrero Avenue, 3D, San Francisco, CA 94II0, USA

Tel + I 4152068823

Fax + I 4155022249

Email Sun-Chuan.Dai@ucsf.edu
Abstract: Gastric neuroendocrine tumors derive from enterochromaffin-like cells in the stomach mucosa. Based on histologic, serologic, and endoscopic findings, they may be further differentiated into types I, II, and III, with varying degrees of aggressiveness. In this article, diagnostic and classification strategies are reviewed, as are endoscopic, systemic, and surgical modalities for management. A multidisciplinary approach is advocated to provide the most effective patient care.

Keywords: neuroendocrine, tumor, carcinoid, gastrin, stomach

\section{Background}

First reported by Askanazy in 1923, gastric neuroendocrine tumors (NETs) comprise approximately $1.8 \%$ of all gastric tumors ${ }^{1}$ and develop from enterochromaffin-like (ECL) cells in the gastric mucosa. Gastric NETs are also referred to as gastric carcinoids. The term karzinoide, or carcinoma-like, was coined by Oberndorfer in 1907 to describe this class of tumors, which behaves in a relatively benign fashion compared to adenocarcinomas. ${ }^{2}$ Our understanding of NETs as a whole has evolved over time, and the World Health Organization (WHO) classification system now employs the term neuroendocrine tumor instead of carcinoid. As such, gastric NETs are part of a broad category of gastroenteropancreatic-neuroendocrine tumors (GEPNETs), which encompass well-differentiated NETs arising from the gastrointestinal tract. In an effort to standardize the system and assist clinicians to accurately predict clinical outcomes, GEPNETs are graded histologically based on mitotic count and/or Ki67 index. The 2010 WHO histologic classification describes well-differentiated NETs as having a Ki67 index $<3 \%$ and $<2$ mitoses per 10 high-power fields (HPFs) (G1), moderately differentiated NETs with a Ki67 index 3-20\% or 2-20 mitoses per $10 \mathrm{HPFs}(\mathrm{G} 2)$, and poorly differentiated NETs with a Ki67 index $>20 \%$ or $>20$ mitoses per $10 \mathrm{HPFs}(\mathrm{G} 3)^{3,4}$

The prevalence of gastric NETs is difficult to establish due to a lack of uniform data collection from cancer registries worldwide. A study published in 2015 analyzing national cancer registries in 10 European countries, the US, and Japan determined the prevalence of gastric NETs per 10,000 population to be 0.32 in Europe, 0.17 in the US, and 0.05 in Japan..$^{5}$ The authors of this study did note that their values may be underestimations due to a tendency of cancer registries to reflect the aggressive tumors that require treatment as opposed to benign tumors.

Clinically, gastric NETs are categorized into types I, II, and III (Table 1). The basis for these subtypes is rooted in gastric pathophysiology. ${ }^{6}$ Gastrin is produced by 
$\mathrm{G}$ cells in the stomach after ingestion of food. Gastrin binds to cholecystokinin-2 receptors on ECL cells, which subsequently release histamine to activate parietal cells to produce hydrochloric acid. Acid production is regulated by negative feedback so that D cells in the antrum produce somatostatin that inhibits $\mathrm{G}$ cell production of gastrin. Therefore, in states where acid production is lacking (achlorhydria), there is compensatory $\mathrm{G}$ cell hyperplasia and hypergastrinemia that leads to ECL cell hyperplasia in an effort to boost acid production. Hypersecretory states with unchecked gastrin production, such as Zollinger-Ellison syndrome (ZES), are also predisposed to ECL cell hyperplasia and NET growth.

Type I and II gastric NETs develop due to hypergastrinemia and ECL cell hyperplasia, while type III gastric NETs arise sporadically. Type I tumors are most common and represent $70-80 \%$ of all gastric NETs. ${ }^{7}$ They tend to be multiple, smaller than $1 \mathrm{~cm}$ in size, and are often discovered incidentally or during endoscopic evaluation for anemia (Figure 1A and B). Their behavior is typically indolent, and metastases are infrequent (occurring in $<10 \%$ of patients for lesions $<2 \mathrm{~cm}$ in size) ${ }^{8}$ Achlorhydric conditions associated with type I gastric NETs include chronic atrophic gastritis (e.g. from type A autoimmune gastritis or vagotomy). Although long-term use of proton pump inhibitor (PPI) leads to chronic hypergastrinemia, there have only been case reports of gastric NETs associated with PPI use. ${ }^{9}$ Type II gastric NETs represent $5-10 \%$ of gastric NETs. ${ }^{7}$ Like type I disease, they are also typically multiple, small, asymptomatic, and well-differentiated tumors. The risk of metastasis is slightly higher in type II gastric NETs

Table I Gastric carcinoid disease categories and characteristics

\begin{tabular}{llll}
\hline Characteristics & Type I & Type II & Type III \\
\hline \% among gastric carcinoids & $70-80 \%$ & $5-10 \%$ & $10-15 \%$ \\
Associated disease & Atrophic gastritis & Gastrinoma & None \\
& Helicobacter pylori & MENI & \\
Gastric pH & High $(>4)$ & Low $(<2)$ & Normal \\
Serum gastrin & High & High & Normal \\
Potential to metastasize & $2-5 \%$ & $10-30 \%$ & $50-100 \%$ \\
\hline
\end{tabular}

compared to type I lesions but is still low. Overall, tumor invasion beyond the submucosa into the muscularis propria or lymph node and liver involvement occurs in 5-12\% of cases. ${ }^{10}$ Type II gastric NETs are also associated with hypergastrinemia, but the feature that distinguishes them from type I tumors is their association with ZES and multiple endocrine neoplasia type 1 (MEN1) syndrome, which are hypersecretory states. Up to $30-50 \%$ of patients with MEN1 syndrome will develop gastric NETs, especially if ZES is present. ${ }^{10}$ Type II gastric NETs unsurprisingly have a high rate of loss of heterogyzosity at the MEN1 gene locus of 75-100\%. Interestingly, 17-73\% of type I gastric NETs and $25-50 \%$ of type III gastric NETs carry the same mutation, suggesting that MEN1 mutations cannot be used to distinguish among the 3 types of gastric NETs. ${ }^{11-13}$ Type II gastric NETs are typically nonfunctioning tumors, and symptoms upon presentation are usually secondary to peptic ulcer disease and ZES. While other genetic syndromes such as type 1 neurofibromatosis, von Hippel-Landau disease, and tuberous sclerosis complex are associated with NETs, gastric involvement is rare.

Approximately $10-15 \%$ of gastric NETs are categorized as type III tumors. These lesions typically exist as solitary larger tumors, often $>2 \mathrm{~cm}$ in size. Histopathologically, they vary from well- to poorly differentiated tumors, and their overall prognosis is relatively poor. Upon initial presentation, the incidence of concurrent metastasis is $>50 \%$, typically with hepatic involvement. Unlike type I and II gastric NETs, type III lesions do not have any associated predisposing conditions. Fasting gastrin levels are normal without any $\mathrm{G}$ cell or ECL cell hyperplasia. While type I and II gastric NETs tend to be nonfunctional tumors, type III NETs with hepatic metastases may be associated with carcinoid syndrome, although this occurrence is rare.

\section{Diagnosis and evaluation}

Studies used to diagnose and differentiate gastric NET disease may be broadly divided into endoscopic, biochemical,
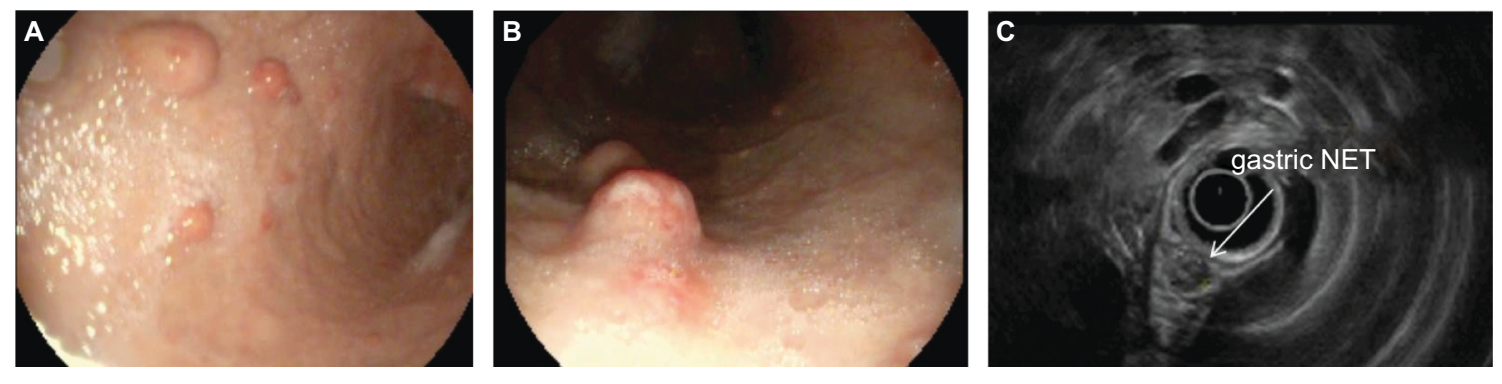

Figure I (A) Endoscopic image of multiple type I gastric neuroendocrine tumors (NETs) in the gastric body. (B) Close-up endoscopic image of a type I gastric NET in the gastric body. (C) Endoscopic ultrasound of a type I gastric NET. The lesion appears hypoechoic and confined to the submucosa, without any invasion of the musclaris propria. 
histopathologic, and imaging studies. As symptoms related to carcinoid syndrome are rare, endoscopy is the gold standard for diagnosing gastric NETs. During esophagoduodenoscopy (EGD), aspiration of gastric fluid may be performed to assess a gastric $\mathrm{pH}$, though this value can be artificially elevated by PPI use. Endoscopic ultrasound (EUS) may be performed on larger lesions to evaluate the depth of gastric wall involvement and for lymphadenopathy. In type I disease, tumors are often found in the gastric fundus and described as subcentimeter and multifocal. Biopsies of the rest of the stomach may detect atrophic gastritis or Helicobacter pylori (HP). Type II disease may also appear as multifocal subcentimeter polypoid lesions, but there may be concurrent peptic ulcer disease in the setting of a hypersecretory state. The gastric $\mathrm{pH}$ is generally high $(>4)$ in type I disease and low $(<2)$ in type II tumors. Tumors in type III disease typically appear as solitary larger lesions that may be ulcerated with hemorrhage. Gastric $\mathrm{pH}$ is typically normal, and there is an association with HP infection but not atrophic gastritis or peptic ulcer disease.

Biochemical testing is often performed to differentiate between the subtypes of gastric NETs. Gastrin levels are elevated in type I and II gastric NETs, while they are normal in type III. However, concurrent or recent PPI use may elevate gastrin levels; therefore, PPIs should be withdrawn at least 1 week before obtaining accurate fasting gastrin levels. ${ }^{14}$ In cases of ZES, abrupt PPI withdrawal can lead to serious consequences, including gastrointestinal perforation, ${ }^{15}$ and a careful PPI wean may be recommended when this entity is suspected. During weaning, PPIs are replaced by histamine H2-receptor antagonists such as ranitidine 1-2 weeks before formal testing. The H2-receptor antagonist should be dosed as $450-750 \mathrm{mg}$ every 6 hours and then stopped in the final 24-30 hours before testing. Antacids may be taken as needed until the midnight before testing. Patients should be given explicit instructions to seek medical attention during this period should they develop diarrhea, nausea, vomiting, or severe abdominal pain. ${ }^{14}$ Further differentiation between type I and II gastric NETs may be established by incorporation of the gastric $\mathrm{pH}$ as gastrin level alone cannot determine type I or type II disease. Finally, given the association of chronic atrophic gastritis with type I gastric NETs, a low serum vitamin b12 and positive parietal cell and/or intrinsic factor antibodies may be found. While genetic testing for MEN1 may be considered when there is suspicion of type II disease, it is not considered a diagnostic tool for gastric NETs. A small case series of type II gastric NET in the setting of a confirmed germline MEN1 mutation has recommended screening for parathyroid and pituitary tumors. ${ }^{16}$
Conventional cross-sectional imaging modalities such as computed tomography (CT) and magnetic resonance imaging (MRI) are commonly used to assess for disease spread and for staging of advanced lesions. However, their value is limited in type I and II diseases as these are frequently characterized by small tumors. Similarly, functional imaging with somatostatin receptor scintigraphy, fluoro-D-glucose positron emission tomography, Ga-DOTATOC PET/CT, or MRI is of limited use in smaller tumors. ${ }^{17,18}$ That said, 68Ga-DOTATOC may be helpful in localizing occult gastrinomas in the setting of type II gastric NETs. ${ }^{19}$ In type III disease where tumors are larger with a tendency to metastasize, conventional CT or MRI studies are helpful for tumor detection and staging. Larger studies are needed to determine the precise role of functional imaging in gastric NETs, though early data on Ga-DOTATOC imaging are promising..$^{20,21}$

\section{Endoscopic management}

Endoscopic management is predominantly utilized in type I and localized type II disease. In type III disease, endoscopy plays a smaller role given the higher likelihood of concurrent metastatic disease. The lack of consensus surrounding the management of gastric NETs is highlighted by discrepancies between the published guidelines of the National Cancer Comprehensive Network (NCCN) and European Neuroendocrine Tumor Society (ENETS). ${ }^{22,23}$ Beyond a conventional EGD, EUS may also be performed to evaluate tumor depth (Figure 1C), though the cutoff size to prompt this procedure is not yet defined. Generally, lesions smaller than $8-10 \mathrm{~mm}$ are not amenable to fine needle biopsy during EUS and lesions smaller than $5 \mathrm{~mm}$ may be challenging to visualize endosonographically. The potential benefits of EUS include determination of any muscularis propria invasion that may preclude a complete endoscopic resection and evaluation of lymphadenopathy. The NCCN recommends EUS in type III disease to evaluate for lymphadenopathy and tumor depth, while an EUS is recommended in type I and II disease as clinically indicated. The ENETS guidelines mention the role of EUS for staging tumors but are less specific with respect to subtype of gastric NETs, and emphasize the need of further investigation to determine the cutoff size for endosonographic examinations of tumors.

In type I gastric NETs, the cutoff size for a tumor to harbor metastatic potential is thought to be $10 \mathrm{~mm} .{ }^{24}$ Assuming there is no muscularis propria or lymph node involvement, endoscopic resection of lesions $>10 \mathrm{~mm}$ is favored in the ENETS guidelines as this is considered the least invasive approach. ${ }^{23}$ The 2017 NCCN guidelines are less specific and 
recommend resection of only "prominent" tumors. ${ }^{22}$ There is no consensus regarding the treatment of subcentimeter lesions, though surveillance annually or every 2 years is favored by both guidelines. The ENETS guidelines recommend performing an EGD with polyp resection or biopsy every 1-2 years. While more aggressive approaches that entail resecting all visible lesions or selectively removing lesions that are at least $5 \mathrm{~mm}$ in size have been described, ${ }^{25,26}$ there have not been any studies comparing these strategies. Recognizing a lack of data from large studies and published series demonstrating tumor recurrence rates of $18-63.6 \%,{ }^{25,26}$ a prudent approach would be to continue endoscopic surveillance even if resection is performed.

EUS has a larger role in type II than type I gastric NETs in order to rule out pancreatic lesions and determine if the gastric tumor is a primary or secondary lesion. Endoscopic resection of a primary type II gastric NET is a feasible primary treatment option according to both NCCN and ENETS guidelines. However, if there are duodenal or pancreatic lesions, a patient-individualized multidisciplinary approach at a neuroendocrine center of expertise may be necessary.

In cases of type III disease in which metastasis has been ruled out, an EUS may assist in evaluating for lymphadenopathy. If negative, endoscopic or surgical resection is recommended, although NCCN guidelines favor endoscopic resection exclusively for superficial subcentimeter lesions with "low-grade" histology. 22

If indicated, endoscopic resection is chiefly accomplished by 2 techniques: endoscopic mucosal resection (EMR) and endoscopic submucosal dissection (ESD). EMR typically involves injecting a saline solution into the submucosal space underneath the lesion to achieve proper lifting and delineation of the lesion's borders, followed by resection with a cauterized snare. ESD requires specialized endoscopic instruments to penetrate a lesion's surrounding submucosal space followed by cautery to complete an en bloc resection. This technique is effective for lesions not thought amenable to EMR and is practiced chiefly in Asia and is less available in Western countries. The literature on ESD for gastric NETs is scant as most publications focus on use of ESD for resection of rectal lesions. Nevertheless, it is considered safe and associated with high rates of complete resection of gastric lesions if done at high-volume centers. EMR and ESD are both associated with a low, but tangible, risk of perforation or bleeding and thus should be performed by an experienced endoscopist. One study from Japan reported on the use of ESD for 42 patients with gastrointestinal NETs (of which most were rectal); there were 2 $(5 \%)$ cases of postoperative bleeding and $2(5 \%)$ perforations. ${ }^{27}$

\section{Medical management}

In type I and II gastric NETs, somatostatin analogs (SSAs) have been shown to decrease levels of gastrin and have an antiproliferative effect on ECL cells. ${ }^{28,29}$ Limited studies, including a few small prospective studies, have demonstrated regression or complete disappearance of tumors and marked decrease in serum gastrin, lasting up to several years. ${ }^{30-39}$ SSAs (e.g. octreotide and lanreotide) can be considered in cases in which endoscopic resection is not feasible due to extensive multifocal disease, or submucosal/lymph node involvement, as well as recurrent disease after repeated endoscopic resection. ${ }^{40}$ However, small studies have shown that this antiproliferative effect is not durable, since a rebound in serum gastrin to pretreatment levels and tumor recurrence are possible after cessation of therapy. ${ }^{35,36,41}$ A repeat cycle of SSA after disease recurrence may again induce tumor regression. ${ }^{41}$ Given the lack of randomized clinical trials with SSAs for this indication, their high cost, and the comparatively benign course of type I/II gastric NETs with endoscopic resection, ENETS recommends their use be restricted to cases of metastatic type I gastric NETs with proven somatostatin receptor 2 (SSTR2) expression and a low $\mathrm{Ki} 67$ index, and NCCN guidelines recommend consideration of SSAs only for type II gastric NET cases in which the primary tumor has not been resected (i.e. to control gastrin secretion), in conjunction with endoscopic surveillance and resection of prominent tumors. ${ }^{22,23,40,42}$ Furthermore, the oral gastrin receptor antagonist netazepide (YF476) is under study as an alternative therapeutic option for patients with type I gastric NETs and has been shown to cause tumor regression and normalize chromogranin A levels in 2 small prospective studies. $^{43,44}$

As in type I and II gastric NETs, systemic therapy in type III is warranted only in locoregional disease that is unresectable or metastatic. Management in these cases is identical to that of all unresectable or metastatic gastrointestinal NETs and is based on a multitude of factors, including the patient's symptoms, tumor grade, tumor burden, and progression of disease during imaging-guided surveillance. ${ }^{22}$ Systemic options for well-differentiated NETs typically include SSAs and everolimus; interferon alfa-2b is less commonly employed. ${ }^{22,45,46}$ Treatment is often multimodal, particularly in cases of liver metastases, for which liver-directed therapies such as hepatic arterial embolization are commonly employed. ${ }^{47,48}$ Peptide receptor radionuclide therapy is an emerging tool for somatostatin receptor-expressing tumors, but is not approved for use in the US. ${ }^{47,48}$ The role of systemic chemotherapy in the treatment of well-differentiated gastric 
NETs is ill-defined. Unfortunately, there are no randomized clinical trials or prospective data comparing the efficacy of one therapy to another, or determining the most efficacious sequence of therapies for well-differentiated NETs. Platinumbased combination chemotherapy is typically reserved for poorly differentiated neuroendocrine carcinomas. ${ }^{22,49-51}$

\section{Surgical management}

Type I gastric NETs are indolent but frequently present with multifocal primary tumors and often recur. ${ }^{6,52}$ However, lymph node metastases are rare $(\sim 10 \%)$, and disease-specific mortality is exceedingly uncommon. ${ }^{53}$ Therefore, careful patient selection for surgery is of paramount importance. Surgical resection of type I gastric NETs may be considered when primary tumors are not amenable to endoscopic resection due to the depth of larger size lesions involving the muscularis propria. Surgery may also be warranted when there is a concern for regional lymph node involvement based on cross-sectional imaging or EUS. Finally, surgery clearly has a role when there is biopsy-proven or suspected coexisting gastric adenocarcinoma arising in the setting of chronic atrophic gastritis. ${ }^{53,54}$

Surgery for type I gastric NETs not amenable to endoscopic resection may entail resection of the primary tumors combined with antrectomy to remove the source of gastrin production. ${ }^{53}$ A regional lymphadenectomy should be done for staging. After antrectomy for type I gastric NETs, gastrin levels typically normalize, and regression of primary tumors occurs in most patients. Recurrent disease may result if a complete antrectomy is not performed and gastrin-producing cells are left behind. ${ }^{53}$ Disease may also recur if gastric NETs develop gastrin-independent growth. Therefore, endoscopic surveillance should be considered after antrectomy to look for recurrent NETs and to screen for gastric adenocarcinoma, which can arise in patients with chronic atrophic gastritis. ${ }^{54}$

Type II gastric NETs arise almost exclusively in patients with a gastrin-producing NET of the duodenum or pancreas (gastrinoma, ZES) and MEN1.6,55,56 In patients with ZES/MEN1, 13-37\% develop type 2 gastric NETs. ${ }^{56}$ Most (80-90\%) of type II gastric NETs are not invasive, and the primary gastrinoma should be resected, if feasible. ${ }^{22,56}$ If resection of the primary gastrinoma is not done, type II gastric NETs can often be managed endoscopically and/or with SSAs. In patients with long-standing hypergastrinemia in the setting of ZES/MEN1, advanced serotonin-producing type II gastric NETs have been observed, requiring total gastrectomy and hepatectomy to control extensive local tumor burden (gastric obstruction) and the carcinoid syndrome, respectively. ${ }^{56}$
Type III gastric NETs may be well differentiated (G1, G2) or poorly differentiated (G3). ${ }^{57}$ Well-differentiated type III gastric NETs are frequently invasive and metastasize to regional lymph nodes; therefore, patients are typically managed with an oncologic resection of the primary tumor and regional lymph nodes. In carefully selected patients with type III gastric NETs with low-risk features $(<2 \mathrm{~cm}$, confined to submucosal layer, no lymphovascular invasion), favorable results with endoscopic resection (EMR, ESD) have been reported in South Korea. ${ }^{58}$ The outcome of patients with poorly differentiated type III gastric NETs is extremely poor, resembling that of patients with small-cell lung cancer; therefore, the role of surgery is quite limited. ${ }^{6}$ A reasonable approach is to treat patients with poorly differentiated type III gastric NETs with upfront platinum-based systemic therapy, and perhaps consider surgical resection in only those who have locoregional disease.

\section{Conclusion}

Gastric NETs are clinically categorized into types I, II, and III. A combination of fasting serum gastrin levels, gastric $\mathrm{pH}$, and endoscopic and imaging findings is necessary to differentiate among the types of gastric NETs as their prognoses all vary. While some gastric NETs are indolent and can be managed by endoscopic resection and surveillance, refractory disease may require treatment with an SSA. Liverdirected therapy and/or systemic therapy with everolimus or chemotherapy is typically reserved for advanced disease. Surgical resection is reserved for type I and type II NETs that are endoscopically unresectable, or carefully selected patients with well-differentiated type III disease. While the NCCN and ENETS provide guidelines, the diagnosis and management of gastric NETs remains challenging in some areas, and a multidisciplinary approach is preferred to ensure consideration of all therapeutic options.

\section{Disclosure}

EKB has received research funding (to institution) from Novartis and served as a consultant (unpaid) for Ipsen. The other authors report no conflicts of interest in this work.

\section{References}

1. Hou W, Schubert ML. Treatment of gastric carcinoids. Curr Treat Options Gastroenterol. 2007;10(2):123-133.

2. Oberndorfer S. Karzinoide tumoren des dunndarms [Carcinoid tumors of the small intestine]. Frank F Z Pathol. 1907;1:426-429. German [with English abstract].

3. Klöppel G, Perren A, Heitz PU. The gastroenteropancreatic neuroendocrine cell system and its tumors: the WHO classification. Ann NY Acad Sci. 2004;1014:13-27. 
4. Bosman FT, Carneiro F, Hruban RH, Theise ND. WHO Classification of Tumours of the Digestive System. 4th ed. Lyon: IARC; 2010.

5. Boyce M, Thomsen L. Gastric neuroendocrine tumors: prevalence in Europe, USA, and Japan, and rationale for treatment with a gastrin/ CCK2 receptor antagonist. Scand J Gastroenterol. 2015;50(5):550-559.

6. Rindi G, Luinetti O, Cornaggia M, Capella C, Solcia E. Three subtypes of gastric argyrophil carcinoid and the gastric neuroendocrine carcinoma: a clinicopathologic study. Gastroenterology. 1993;104(4):994-1006.

7. Borch K, Ahrén B, Ahlman H, Falkmer S, Granérus G, Grimelius L. Gastric carcinoids: biologic behavior and prognosis after differentiated treatment in relation to type. Ann Surg. 2005;242(1):64-73.

8. Rindi G, Bordi C, Rappel S, Rosa SL, Stolte M, Solcia E. Gastric carcinoids and neuroendocrine carcinomas: pathogenesis, pathology, and behavior. World J Surg. 1996;20(2):168-172.

9. Nandy N, Hanson JA, Strickland RG, McCarthy DM. Solitary gastric carcinoid tumor associated with long-term use of omeprazole: a case report and review of the literatures. Dig Dis Sci. 2016;61(3):708-712.

10. Basuroy R, Srirajaskanthan R, Prachalias A, Quaglia A, Ramage JK. Review article: the investigation and management of gastric neuroendocrine tumors. Aliment Pharmacol Ther. 2014;39(10):1071-1084.

11. Bordi C. Neuroendocrine pathology of the stomach: the Parma contribution. Endocr Pathol. 2014;25(2):171-180.

12. Debelenko LV, Emmert-Buck MR, Zhuang Z, et al. The multiple endocrine neoplasia type I gene locus is involved in the pathogenesis of type II gastric carcinoids. Gastroenterology. 1997;113(3):773-781.

13. Modlin IM, Lye KD, Kidd M. Carcinoid tumors of the stomach. Surg Oncol. 2003;12(2):153-172.

14. Metz DC. Diagnosis of the Zollinger Ellison syndrome. Clin Gastroenterol Hepatol. 2012;10(2):126-130.

15. Poitras P, Gingras MH, Rehfeld JF. The Zollinger-Ellison syndrome: dangers and consequences of interrupting antisecretory treatment. Clin Gastroenterol Hepatol. 2012;10(2):199-202.

16. Nikou GC, Toubanakis C, Nikolaou P, et al. Gastrinomas associated with MEN-1 syndrome: new insights for the diagnosis and management in a series of 11 patients. Hepatogastroenterology. 2005;52(66):1668-1676.

17. Beiderwellen KJ, Poeppel TD, Hartung-Knemeyer V, et al. Simultaneous 68Ga-DOTATOC PET/MRI in patients with gastroenteropancreatic neuroendocrine tumors: initial results. Invest Radiol. 2013;48(5):273-279.

18. Cavallaro A, Zanghi A, Cavallaro M, et al. The role of 68-GA-DOTATOC CT-PET in surgical tactic for gastric neuroendocrine tumors treatment: our experience: a case report. Int J Surg. 2014;12 Suppl 1: S225-S231.

19. Naswa N, Sharma P, Soundararajan R, et al. Diagnostic performance of somatostatin receptor PET/CT using ${ }^{68} \mathrm{Ga}$-DOTANOC in gastrinoma patients with negative or equivocal CT findings. Abdom Imaging. 2013;38:552-560.

20. Hope TA, Pampaloni MH, Nakakura E, et al. Simultaneous (68) Ga-DOTA-TOC PET/MRI with gadoxetate disodium in patients with neuroendocrine tumor. Abdom Imaging. 2015;40(6):1432-1440.

21. Menda Y, O'Dorisio TM, Howe JR, et al. Localization of unknown primary site with ${ }^{68} \mathrm{Ga}$-DOTATOC PET/CT in patients with metastatic neuroendocrine tumor. $J$ Nucl Med. 2017;58(7):1054-1057.

22. Kulke MH, Shah MH, Benson AB, et al. National Comprehensive Cancer Network. Neuroendocrine tumors, version 3.2017. Available from: www.nccn.org. Accessed June 13, 2017.

23. Delle Fave G, O’Toole D, Sundin A, et al; Vienna Consensus Conference participants. ENETS consensus guidelines update for gastroduodenal neuroendocrine neoplasms. Neuroendocrinology. 2016;103(2):119-124.

24. Gorzinsky-Glasberg S, Thomas D, Strosberg JR, et al. Metastatic type 1 gastric carcinoid: a real threat or just a myth? World J Gastroenterol. 2013;19(46):8687-8695.

25. Merola E, Sbrozzi-Vanni A, Panzuto F, et al. Type I gastric carcinoids: a prospective study on endoscopic management and recurrence rate. Neuroendocrinology. 2012;95(3):207-213.

26. Uygun A, Kadayifci A, Polat Z, et al. Long-term results of endoscopic resection for type I gastric neuroendocrine tumors. J Surg Oncol. 2014;109:71-74.
27. Suzuki S, Ishii N, Uemura M, et al. Endoscopic submucosal dissection (ESD) for gastrointestinal carcinoid tumors. Surg Endosc. 2012;26(3):759-763.

28. Burkitt MD, Varro A, Pritchard DM. Importance of gastrin in the pathogenesis and treatment of gastric tumors. World J Gastroenterol. 2009;15(1):1-16.

29. Sato Y, Hashimoto S, Mizuno K, Takeuchi M, Terai S. Management of gastric and duodenal neuroendocrine tumors. World J Gastroenterol. 2016;22(30):6817-6828.

30. Campana D, Nori F, Pezzilli R, et al. Gastric endocrine tumors type I: treatment with long-acting somatostatin analogs. Endocr Relat Cancer. 2008;15(1):337-342.

31. Campana D, Ravizza D, Ferolla P, et al. Clinical management of patients with gastric neuroendocrine neoplasms associated with chronic atrophic gastritis: a retrospective, multicentre study. Endocrine. 2016;51(1):131-139.

32. Dakin GF, Warner RR, Pomp A, Salky B, Inabnet WB. Presentation, treatment, and outcome of type 1 gastric carcinoid tumors. J Surg Oncol. 2006;93(5):368-372.

33. Fykse V, Sandvik AK, Qvigstad G, Falkmer SE, Syversen U, Waldum HL. Treatment of ECL cell carcinoids with octreotide LAR. Scand J Gastroenterol. 2004;39(7):621-628.

34. Grozinsky-Glasberg S, Kaltsas G, Gur C, et al. Long-acting somatostatin analogues are an effective treatment for type 1 gastric carcinoid tumours. Eur J Endocrinol. 2008;159(4):475-482.

35. Jianu CS, Fossmark R, Syversen U, Hauso Ø, Fykse V, Waldum HL. Five-year follow-up of patients treated for 1 year with octreotide long-acting release for enterochromaffin-like cell carcinoids. Scand J Gastroenterol. 2011;46(4):456-463.

36. Khuroo MS, Khuroo MS, Khuroo NS. Treatment of type I gastric neuroendocrine tumors with somatostatin analogs. $J$ Gastroenterol Hepatol. 2010;25(3):548-554.

37. Manfredi S, Pagenault M, de Lajarte-Thirouard AS, Bretagne JF. Type 1 and 2 gastric carcinoid tumors: long-term follow-up of the efficacy of treatment with a slow-release somatostatin analogue. Eur J Gastroenterol Hepatol. 2007;19(11):1021-1025.

38. Thomas D, Tsolakis AV, Grozinsky-Glasberg S, et al. Long-term followup of a large series of patients with type 1 gastric carcinoid tumors: data from a multicenter study. Eur J Endocrinol. 2013;168(2):185-193.

39. Tomassetti P, Migliori M, Caletti GC, Fusaroli P, Corinaldesi R, Gullo L. Treatment of type II gastric carcinoid tumors with somatostatin analogues. $N$ Engl J Med. 2000;343(8):551-554.

40. Massironi S, Zilli A, Conte D. Somatostatin analogs for gastric carcinoids: for many, but not all. World J Gastroenterol. 2015;21(22): 6785-6793.

41. Massironi S, Zilli A, Fanetti I, Ciafardini C, Conte D, Peracchi M. Intermittent treatment of recurrent type-1 gastric carcinoids with somatostatin analogues in patients with chronic autoimmune atrophic gastritis. Dig Liver Dis. 2015;47(11):978-983.

42. Massironi S, Sciola V, Spampatti MP, Peracchi M, Conte D. Gastric carcinoids: between underestimation and overtreatment. World J Gastroenterol. 2009;15(18):2177-2183.

43. Fossmark R, Sørdal Ø, Jianu CS, et al. Treatment of gastric carcinoids type 1 with the gastrin receptor antagonist netazepide (YF476) results in regression of tumours and normalisation of serum chromogranin A. Aliment Pharmacol Ther. 2012;36(11-12):1067-1075.

44. Moore AR, Boyce M, Steele IA, Campbell F, Varro A, Pritchard DM. Netazepide, a gastrin receptor antagonist, normalises tumour biomarkers and causes regression of type 1 gastric neuroendocrine tumours in a nonrandomised trial of patients with chronic atrophic gastritis. PLoS One. 2013;8(10):76462.

45. Granberg D, Wilander E, Stridsberg M, Granerus G, Skogseid B, Oberg $\mathrm{K}$. Clinical symptoms, hormone profiles, treatment, and prognosis in patients with gastric carcinoids. Gut. 1998;43(2):223-228.

46. Kim SY, Woo IS, Yang JH, Han CW, Roh SY, Jung YH. A case of metastatic gastric neuroendocrine tumor: therapeutic considerations. Case Rep Oncol. 2014;7(1):266-272. 
47. Caplin ME, Hodgson HJ, Dhillon AP, et al. Multimodality treatment for gastric carcinoid tumor with liver metastases. Am J Gastroenterol. 1998;93(10):1945-1948.

48. Nikou GC, Angelopoulos TP. Current concepts on gastric carcinoid tumors. Gastroenterol Res Pract. 2012;2012:287825.

49. Bongiovanni A, Riva N, Ricci M, et al. First-line chemotherapy in patients with metastatic gastroenteropancreatic neuroendocrine carcinoma. Onco Targets Ther. 2015;8:3613-3619.

50. Okita NT, Kato K, Takahari D, et al. Neuroendocrine tumors of the stomach: chemotherapy with cisplatin plus irinotecan is effective for gastric poorly-differentiated neuroendocrine carcinoma. Gastric Cancer. 2011;14(2):161-165.

51. Yamaguchi T, Machida N, Morizane C, et al. Multicenter retrospective analysis of systemic chemotherapy for advanced neuroendocrine carcinoma of the digestive system. Cancer Sci. 2014;105(9):1176-1181.

52. Postlewait LM, Baptiste GG, Ethun CG, et al. A 15-year experience with gastric neuroendocrine tumors: does type make a difference? J Surg Oncol. 2016;114(5):576-580.
53. Gladdy RA, Strong VE, Coit D, et al. Defining surgical indications for type I gastric carcinoid tumor. Ann Surg Oncol. 2009;16(11):3154-3160.

54. Lahner E, Esposito G, Pilozzi E, et al. Occurrence of gastric cancer and carcinoids in atrophic gastritis during prospective long-term follow up. Scand J Gastroenterol. 2015;50(7):856-865.

55. Berna MJ, Annibale B, Marignani M, et al. A prospective study of gastric carcinoids and enterochromaffin-like cell changes in multiple endocrine neoplasia type 1 and Zollinger-Ellison syndrome: identification of risk factors. J Clin Endocrinol Metab. 2008;93(5):1582-1591.

56. Norton JA, Melcher ML, Gibril F, Jensen RT. Gastric carcinoid tumors in multiple endocrine neoplasia-1 patients with Zollinger-Ellison syndrome can be symptomatic, demonstrate aggressive growth, and require surgical treatment. Surgery. 2004;136(6):1267-1274.

57. Kidd M, Gustafsson B, Modlin IM. Gastric carcinoids (neuroendocrine neoplasms). Gastroenterol Clin North Am. 2013;42(2):381-397.

58. Kwon YH, Jeon SW, Kim GH, et al. Long-term follow up of endoscopic resection for type 3 gastric NET. World J Gastroenterol. 2013;19(46):8703-8708.
Gastrointestinal Cancer: Targets and Therapy

\section{Publish your work in this journal}

Gastrointestinal Cancer: Targets and Therapy is an international, peer-reviewed, open access journal focusing on gastro-intestinal cancer research, identification of therapeutic targets and the optimal use of preventative and integrated treatment interventions to achieve improved outcomes, enhanced survival and quality of life for the

\section{Dovepress}

cancer patient. The manuscript management system is completely online and includes a very quick and fair peer-review system. Visit http://www.dovepress.com/testimonials.php to read real quotes from published authors.

Submit your manuscript here: https://www.dovepress.com/gastro-intestinal-cancer-targets-and-therapy-journal 